Erratum

\title{
SAXAGLIPTIN LEVELS AND ITS PHARMACOKINETIC APPLICATION IN PRESENCE OF SUCRALOSE IN ANIMALS SERUM BY HPLC METHOD: A RESEARCH ARTICLE
}

\author{
WAEL ABU DAYYIH ${ }^{* 1}$, LINA TAMIMI ${ }^{1}$, EYAD MALLAH ${ }^{1}$, KENZA MANSOUR ${ }^{1}$, TAWFIQ ARAFAT ${ }^{1}$, MONA BUSTAMI ${ }^{2}$
}

1Department of Pharmaceutical Medicinal Chemistry and Pharmacognosy, Faculty of Pharmacy and Medical Sciences-University of Petra, Jordan, ${ }^{2}$ Department of Pharmacology and Biomedical Sciences

Email: wabudayyih@uop.edu.jo

Ref: http://www.ijppsjournal.com/Vol7Issue9/6109.pdf

\section{ABSTRACT}

Objective: It is to develop a simple, valid and rapid chromatographic method for quantification of saxagliptin in rat's serum in order to study saxagliptin pharmacokinetic parameters in sucralose fed rats simultaneously to detect any interaction possibility between saxagliptin and sucralose in rats.

Methods: In our developed method of analysis, mobile phase consisted of phosphate buffer $(\mathrm{pH}=4)$ and methanol $(70: 30) \mathrm{v} / \mathrm{v}$ at flow rate of 1 $\mathrm{ml} / \mathrm{min}$ with UV detection at $230 \mathrm{~nm}$., C8 column of separation was used with temperature of $40 \mathrm{C}{ }^{\circ}$ using injection volume of $50 \mu \mathrm{l}$, samples run time was $10 \mathrm{~min}$, and sildenafil citrate was used as internal standard. saxagliptin was given to rats orally of $(2 \mathrm{~g} / \mathrm{kg})$ dose while sucralose was given with $(11 \mathrm{mg} / \mathrm{kg} /$ day $)$ dose.

Results: A successful HPLC method was validated and developed to determine saxagliptin in rats serum, overall intra-day precision and accuracy were reasonable with coefficient of variation percentage CV \% values range (o.14-4.03) and accuracy \% range (99.5-104), while inter-day precision and accuracy showed accepted precision with CV\% range (0.15-2.81) and accuracy \% range (99.9-116). The coefficient of correlation was 0.99949 with reasonable sensitivity and selectivity. Combination effect of saxagliptin with sucralose on saxagliptin serum profile was demonstrated as strong statistical effect according to Cohen's $d$ and significant $P$ values too.

Conclusion: A successful HPLC method was validated and developed to quantify saxagliptin in rats serum, combination effect of saxagliptin with sucralose over all time intervals of saxagliptin serum profile was demonstrated as strong statistical effect.

Keywords: HPLC, Saxagliptin, Sucralose, Pharmacokinetic, Interaction.

The article has a wrong part of introduction by mistake of authors. On request to remove the wrong part of this article and which by mistake we put another introduction for another drug place in place will be read as for another drug, of the published article. Reason is that a big mistake because we talk about Saxagliptin in the article but the introduction is about Pioglitazone.

This part must be removed urgently (in manuscript)

Saxagliptin: (( \pm )-5-(4-(2-(5-ethyl-2-pyridinyl) ethoxy) phenyl) methyl)-2, 4-) thiazolidinedione monohydrochloride [1], the structural formula is as shown in fig. 1. It belongs to a different chemical class with different pharmacological action than the sulfonylureas, metformin, or the $\alpha$-glucosidase inhibitors [2], it is a compound that belongs to a group named "thiazolidinediones" family, an oral antidiabetic agent that acts by decreasing insulin resistance [3]. Saxagliptin has the same mechanism of action by which all thiazolidinediones act inside the body.

Its mechanism of action made it as one of the most effective drugs that is used in the management of type 2 diabetes mellitus [4, 5], saxagliptin can improve the sensitivity to insulin in muscle and adipose tissue $[2,7]$ which helps in hepatic gluconeogenesis inhibition [8] and improves glycemic control by reducing circulating insulin levels $[9,10]$. Type 2 diabetes patients are usually treated with numerous pharmacological compounds which increases the susceptibility to be exposed to risky drug-drug interactions [7].
Saxagliptin was marketed at USA in 1999, nowadays; it is marketed in more than 40 countries worldwide [6].

And the following part must substitutes (in the place of removed one)

Saxagliptin HCL is one of a new generation of orally hypo-glycemic (anti-diabetic medication) from the dipeptidyl peptidase-4 inhibitor class of type 2 drugs for the treatment of diabetic (1-5). Saxagliptin one of a huge class of diabetes mellitus drugs known as dipeptidyl peptidase-4 (DPP-4) inhibitors. This DPP-4 is an enzyme that breaks down incretin hormones. As a DPP-4 inhibitor (3-6), Saxagliptin slows down the breakdown of incretin hormones, increasing the level of these hormones in the body. It is this increase in incretin hormones that is responsible for the beneficial actions of X-gliptins, including increasing insulin level production in answering to meals and decreasing the amount of glucose that the liver organ produces.

Because incretin hormones are more active in response to higher blood glucose/sugar levels, the risk of dangerously low blood sugar (hypoglycaemia) is low with Saxagliptin. Saxagliptin is available as tablets at the dose of 5 and $10 \mathrm{mg}$ in the market under the brand name of Onglyza ${ }^{\circledR}$, by and Astra-Zeneca in Jordan Market. Chemically (1S, 3S, 5S)-2-[(2S)-Amino (3-hydroxytricyclo [3.3.1.13, 7] dec-1-yl) acetyl]-2-azabicyclo [3.1.0] hexane-3-carbonitrile mono-hydrochloride with empirical formula is $\mathrm{C}_{18} \mathrm{H}_{25} \mathrm{~N}_{3} \mathrm{O}_{2}$. $\mathrm{HCl}(1-10)$. 
ABSTRACT

Objective: It is to develop a simple, valid and rapid chromatographic method for quantification saxagliptin pharmacokinetics parameters in sucralose fed rats simultaneously to detect any in sucralose in rats.

Methods: In our developed method of analysis, mobile phase was consisted of phosphate buffer (pH $\mathrm{ml} / \mathrm{min}$ with UV detection at $230 \mathrm{~nm}$. C8 column of separation was used with the temperature of 40 time was $10 \mathrm{~min}$, and sildenafil citrate was used as internal standard. Saxagliptin was given to rats of with $(11 \mathrm{mg} / \mathrm{kg} /$ day $)$ dose.

Results: A successful HPLC method was validated and developed to determine saxagliptin in rats se were reasonable with coefficient of variation percentage CV \% values range $(0.14-4.03)$ and accuracy and accuracy showed accepted precision with CV\% range $(0.15-2.81)$ and accuracy $\%$ range $(99.9-1)$ with reasonable sensitivity and selectivity. Combination effect of saxagliptin with sucralose on sa: strong statistical effect according to Cohen's $d$ and significant $P$ values too.

Conclusion: A successful HPLC method was validated and developed to quantify saxagliptin in rats sucralose over all time intervals of saxagliptin serum profile was demonstrated as strong statistical effi Keywords: HPLC, Saxagliptin, Sucralose, Pharmacokinetic, Interaction.

\section{INTRODUCTION}

Saxagliptin: (( \pm$)-5-(4-(2-(5-$ ethyl-2-pyridinyl) ethoxy) phenyl) methyl)-2, 4-) thiazolidinedione monohydrochloride [1], the structural formula is as shown in fig 1 . It belongs to a different chemical class with different pharmacological action than the sulfonylureas, metformin, or the $\alpha$-glucosidase inhibitors [2], it is a compound that belongs to a group named "thiazolidinediones" family, an oral antidiabetic agent that acts by decreasing insulin resistance [3]. Saxagliptin has the same mechanism of action by which all thiazolidinediones act inside the body. Its mechanism of action made it as one of the most effective drugs that is used in the management of type 2 diabetes mellitus $[4,5]$. saxagliptin can improve the sensitivity to insulin in muscle and adipose tissue $[2,7]$ which helps in hepatic gluconeogenesis inhibition $[8]$ and improves glycemic control by reducing circulating insulin levels $[9,10]$.

Type 2 diabetes patients are usually treated with numerous pharmacological compounds which increases the susceptibility to be

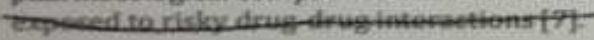

Saxagliptin was marketed at USA in 1999, now a days; it is marketed in more than 40 countries world wide [6].
Sucralose is a synthet formula of Dichlorodeosy $\alpha$-D-galactopyran fig. 2 . It is considered a world's food industries

Sucralose is a derivativ aromatic flavor and goo per $1 \mathrm{gm})$, there are concerning pharmacol potential for sweeter studies have approved $t$ P-glycoprotein intestina activity in intestine an associated with reduc pharmacodynamic parar be metabolized by CYP sucralose at doses that fustify the need of furth drug interactions. 